\title{
Accessibility percolation in random fitness landscapes
}

\author{
Joachim Krug
}

\begin{abstract}
The fitness landscape encodes the mapping of genotypes to fitness and provides a succinct representation of possible trajectories followed by an evolving population. Evolutionary accessibility is quantified by the existence of fitnessmonotonic paths connecting far away genotypes. Studies of accessibility percolation use probabilistic fitness landscape models to explore the emergence of such paths as a function of the initial fitness, the parameters of the landscape or the structure of the genotype graph. This chapter reviews these studies and discusses their implications for the predictability of evolutionary processes.
\end{abstract}

Determining the paths that evolution does not take is as important in evolutionary outcomes as shaping those it may pass through.

T.N. Starr and J.W. Thornton 63]

\section{Introduction}

An evolving population traces out a path in the space of genetic sequences or genotypes. Depending on the level of resolution, the genotype may be described in terms of nucleotide bases, amino acid residues or the alleles of genes. It has been recognised for a long time that sequence spaces are vast, and that only a tiny fraction of all sequences code for viable phenotypes. This raises the question of how evolution nevertheless manages to navigate these spaces across macro-evolutionary distances [3, 37].

An early mathematical formulation of this problem was presented by John Maynard Smith, who estimated the fraction of functional proteins from a percolation argument [43. Assuming that evolution is restricted to proceed by single amino acid substitutions, he conceptualised the space of all protein sequences as a network where each protein is connected to its $n$ one-step mutant neighbours, and a fraction $p$ of proteins is functional. The expected number of functional neighbours of a given sequence is therefore $p n$. It is then plausible (and can be proved [24, 56, 57]) that a large connected component of functional proteins exists with high probability if $p n>1$. Since typically $n \sim 10^{3}$, only a small fraction of proteins has to be functional to ensure evolvability over large genetic distances.

In this chapter we introduce and review a different kind of percolation problem motivated by evolutionary adaptation. In its most general form the problem of accessibility percolation can be formulated as follows [46]. Consider a graph $G=(V, E)$ where each vertex $x \in V$ is labelled by a real-valued random number $f_{x}$ drawn from a joint continuous distribution. We call a path between two vertices $x, y$ accessible if 
the random numbers along the path are monotonically increasing \pm , and we ask for the probability of existence of such a path when the distance $d(x, y)$ between the two points (and the size of the graph) becomes large. The use of the term percolation in this context is motivated by the fact that one studies a certain connectivity property of a random structure. A more specific relation to conventional forms of percolation will be described below in Sect. 2.1.

The notion of evolutionary accessibility was first introduced by Daniel Weinreich and collaborators [67]. In a seminal empirical study they constructed and characterised all $2^{5}=32$ combinations of 5 point mutations in the bacterial antibiotic resistance gene TEM-1 $\beta$-lactamase [68. TEM-1 confers resistance to ampicillin but has very low activity against the novel antibiotic cefotaxime. In combination, the 5 point mutations in TEM-1 increase the baseline resistance against cefotaxime (measured in terms of the concentration up to which bacterial growth is possible) by a factor of about $10^{5}$. The aim of the study was to reconstruct the mutational pathways along which the highly resistant mutant could arise, assuming that mutations occur one at a time and that every step has to provide a benefit in terms of increased resistances. Since each path corresponds to a particular order of occurrence of the mutational steps, there are 5 ! = 120 distinct paths, of which only 18 were found to be accessible. This observation lead the authors to conclude that adaptive evolution is more constrained, and hence more predictable, than previously appreciated.

Along with other related empirical studies [38, 55], the work of Weinreich et al. motivated the first theoretical investigations of evolutionary accessibility in random fitness landscapes [10, 22]. Here the term fitness landscape refers to the assignment of fitness values to genotypes that are connected by mutations [16, 17, 20, 62, 64]. In terms of the general definition of accessibility percolation given above, the graph of interest in this case is the space of genetic sequences endowed with the standard Hamming metric, and the random labels encode fitness or some proxy thereof, such as antibiotic resistance. The precise mathematical setting will be introduced in the next section, and the basic phenomenology will be explained with particular emphasis on the occurrence of abrupt, percolation-like transitions in the accessibility properties. Section 3 reviews accessibility percolation on trees, which sheds additional light on the role of graph geometry, and some generalisations of the standard models will be discussed in Sections 4 and 5, Concluding remarks addressing the relation between accessibility and predictability as well as the role of accessible paths in the evolutionary dynamics are presented in Sect. 6. In addition to a survey of the literature, some unpublished new results are reported in Sections 2.3 and 5. In its focus on the structure of fitness landscapes, the chapter is complementary to the contributions of Anton Bovier [7] and Wolfgang König [35] to this volume, where different types of evolutionary dynamics on fitness landscapes are discussed.

$\ddagger$ The requirement of strict monotonicity will be relaxed in Sect. 5 .

$\S$ The conditions for these assumptions to hold are summarised in Sect. 6.2. 

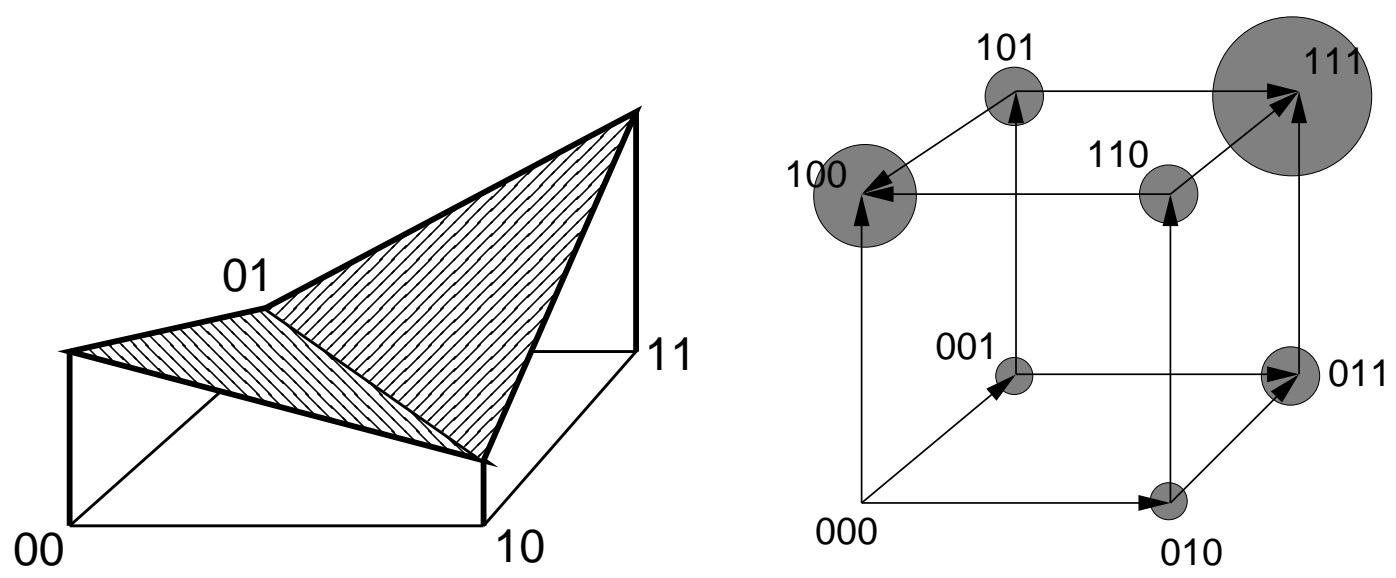

Figure 1. Fitness landscapes on the binary hypercube of dimension $L=2$ (left) and $L=3$ (right) loci. In the left panel the fitness values are plotted on the vertical axes, whereas in the right panel they are represented by circles of different size, and arrows on the edges point in the direction of higher fitness. The fitness landscape on the left is double-peaked, and as a consequence there are no accessible paths from $x=00$ to $y=11$. In the right panel 4 out of $6=3$ ! possible directed paths from 000 to 111 are accessible.

\section{Accessibility percolation in sequence space}

Genotypes are encoded by sequences $x$ of length $L$ with entries drawn from an alphabet $\mathcal{A}=\{0,1,2, . ., a-1\}$ of size $|\mathcal{A}|=a$. The elements of $\mathcal{A}$ will be referred to as alleles. The Hamming distance between two genotypes $x, y$ is defined by

$$
d(x, y)=\sum_{i=1}^{L}\left(1-\delta_{\tau_{i}(x), \tau_{i}(y)}\right),
$$

where $\tau_{i}(x) \in \mathcal{A}$ is the $i$ 'th entry of $x$, that is, the allele at the $i$ 'th genetic locus. Loci are elements of the locus set $\mathcal{L}=\{1,2, \cdots, L\}$ with $|\mathcal{L}|=L$. The sequence space $\mathcal{A}^{L}$ endowed with the Hamming metric $d(\cdot, \cdot)$ is the Hamming graph $\mathbb{H}_{a}^{L}$ [62]. The binary or biallelic Hamming graphs $\mathbb{H}_{2}^{L}$ are $L$-dimensional hypercubes, see Fig. 1 for illustration. Fitness values $f_{x}$ are drawn from a continuous distribution and assigned independentlyt to genotypes [31]. Since accessibility depends only on the rank ordering of genotypes, the distribution of fitness values does not need to be specified. In the following we assume without loss of generality that the $f_{x}$ are uniformly distributed on $[0,1]$. The fitness values induce a natural orientation of the Hamming graph where links between neighbouring sequences are oriented in the direction of increasing fitness. The resulting acyclic directed graph is called the fitness graph and provides a convenient visualisation of the accessible paths [12, 15], see Fig. 11 for illustration.

The quantity of main interest in this chapter is the number of accessible evolutionary paths between two genotypes $x, y$ with $f_{y}>f_{x}$. Throughout we will denote this integer-valued, non-negative random variable by $X_{x, y}$. While it would be desirable to

$\dagger$ The assumption of independence will be dropped in Section 4 . 
characterise the full distribution of $X_{x, y}$, we will mostly be restricted to statements about the expectation $\mathbb{E}\left(X_{x, y}\right)$ and the probability of existence of at least one path $\mathbb{P}\left(X_{x, y} \geq 1\right)$, where both quantities are understood to be conditioned on some positive value of the fitness difference $f_{y}-f_{x}$. Colloquially, we will sometimes say that a genotype space is accessible (inaccessible) if $\mathbb{P}\left(X_{x, y} \geq 1\right) \rightarrow 1\left(\mathbb{P}\left(X_{x, y} \geq 1\right) \rightarrow 0\right)$ when $d(x, y) \rightarrow \infty[22$.

\subsection{Directed paths}

A path of length $\ell$ between two genotypes $x, y$ is a sequence

$$
x \rightarrow x_{1} \rightarrow x_{2} \rightarrow \cdots \rightarrow x_{\ell-1} \rightarrow y
$$

of genotypes such that $d\left(x, x_{1}\right)=d\left(x_{i}, x_{i+1}\right)=d\left(x_{\ell-1}, y\right)=1$ for $i=1, \cdots, \ell-1$, and it is accessible if

$$
f_{x}<f_{x_{1}}<f_{x_{2}}<\cdots<f_{x_{\ell-1}}<f_{y} .
$$

Along a directed path the distance to the target genotype $y$ decreases by one in each step, which implies that $\ell=d(x, y)$ and $d\left(x, x_{i}\right)=i$. The properties of directed paths do not depend on the number of alleles $a$, since the only mutations that occur along such a path convert an allele of the initial genotype $x$ into the corresponding allele of the final genotype $y$ [69]. There are $\ell$ such mutations that can occur in arbitrary order, hence the total number of directed paths is $\ell$ !.

We begin by computing the expected number of accessible directed paths conditioned on the fitness difference $\beta=f_{y}-f_{x}>0$. The condition (3) implies that all intermediate fitness values must lie between $f_{x}$ and $f_{y}$, which happens with probability $\beta^{\ell-1}$, and additionally they have to be ordered, which happens with probability $\frac{1}{(\ell-1) !}$. Thus the probability for the path to be accessible is

$$
P_{\beta, l}=\frac{\beta^{\ell-1}}{(\ell-1) !} .
$$

This expression suggests an alternative interpretation which emphasises the link to percolation. Suppose we fix the initial and final fitness values at $f_{x}=0$ and $f_{y}=1$, and remove all other genotypes (endowed with i.i.d. fitness values) independently with probability $1-\beta$. Then the joint probability for a path of length $\ell$ from $x$ to $y$ to exist and to be accessible is precisely given by (4), and $\beta$ is seen to play the role of the occupation probability in a standard percolation problem.

Multiplying (4) with the total number of directed paths we arrive at [26]

$$
\mathbb{E}_{\ell, \beta}\left(X_{x, y}\right)=\ell ! \frac{\beta^{\ell-1}}{(\ell-1) !}=\ell \beta^{\ell-1} .
$$

For any fixed $\beta<1$, the expectation tends to zero fon $₫ \rightarrow \infty$, and by Markov's inequality we conclude that $\lim _{\ell \rightarrow \infty} \mathbb{P}_{\ell, \beta}\left(X_{x, y} \geq 1\right)=0$ as well. If $\beta$ is allowed to vary with $\ell$ as $\beta_{\ell}=1-\epsilon_{\ell}$ such that $\epsilon_{\ell} \rightarrow 0$, the expectation (5) vanishes in the limit if

$\ddagger$ Here and in the following the limit $\ell \rightarrow \infty$ is understood to be taken along with the limit $L \rightarrow \infty$. In the case of directed paths we can set $\ell=L$ without loss of generality. 
$\epsilon_{\ell}>\frac{\ln \ell}{\ell}$ and diverges if $\epsilon_{\ell}<\frac{\ln \ell}{\ell}$. In the latter case no statement about the existence of accessible paths can be inferred from Markov's inequality. Hegarty and Martinsson [26] used the second moment inequality [1]

$$
\mathbb{P}\left(X_{x, y} \geq 1\right) \geq \frac{\mathbb{E}\left(X_{x, y}\right)^{2}}{\mathbb{E}\left(X_{x, y}^{2}\right)}
$$

to show that the upper bound provided by the first moment is essentially tight, in the sense that

$$
\lim _{\ell \rightarrow \infty} \mathbb{P}_{\ell, \beta_{\ell}}\left(X_{x, y} \geq 1\right)= \begin{cases}0 & : \quad \epsilon_{\ell}=1-\beta_{\ell}>\frac{\ln \ell}{\ell}+\delta_{\ell} \\ 1 & : \quad \epsilon_{\ell}=1-\beta_{\ell}<\frac{\ln \ell}{\ell}-\delta_{\ell}\end{cases}
$$

where $\delta_{\ell}>0$ with $\lim _{\ell \rightarrow \infty} \ell \delta_{\ell}=\infty$. For the estimate of the second moment in (6) one needs to consider pairs of paths and their intersections.

Thus for directed paths a transition from low to high accessibility occurs near $\beta=1$, and this result can be read off from the behaviour of the expectation $\mathbb{E}(X)$. The full distribution of the number of accessible directed paths was obtained by Berestycki, Brunet and Shi [5]. Working in a scaling limit where $\epsilon_{\ell}=\frac{C}{\ell}$ for $\ell \rightarrow \infty$ with $C>0$, they show that $X_{x, y} / \ell$ converges in law to $\exp (-C)$ times the product of two standard independent exponential random variables.

The result (7) can also be applied to the setting originally considered in [22], where the paths were constrained to end at the global fitness maximum (which corresponds to setting $f_{y}=1$ ) but the initial fitness was not specified. This amounts to integrating (5) with respect to $\beta=1-f_{x}$. Remarkably, the result

$$
\int_{0}^{1} d \beta \mathbb{E}\left(X_{\ell, \beta}\right)=1
$$

is independent of $\ell$ and thus illustrates the fact that the directed hypercube is "marginally" accessible. Whereas (8) could naively be interpreted to imply that accessible paths are likely to exist, the simulations reported in [22] showed that most realisations of landscapes had $X_{x, y}=0$, and the unit mean value was achieved through rare instances with $X_{x, y} \gg 1$. On the basis of (17) these instances are understood to be those where the initial fitness happens to be below $\frac{\ln \ell}{\ell}$.

\subsection{Paths with back steps}

From a biological perspective there is no good reason to exclude the possibility of mutational reversions, where a mutation that occurs at some point along the path is later reverted. Specialising to the biallelic case with $a=2$, a path between two genotypes at distance $D$ that includes $k$ reversions has total length $\ell=D+2 k$, since each reversion has to be compensated by an additional forward step. When mutations are rare, evolution is expected to proceed preferentially along the shortest paths, and longer paths are obviously also less likely to be accessible. This disadvantage may however be offset by the enormous increase in the number of possible paths, which merely have to be self-avoiding. A re-analysis of the 5-dimensional TEM-1 $\beta$-lactamase 
resistance landscape of Weinreich et al. 68] that included mutational reversions found a moderate increase in the number of accessible paths, from 18 to 27 [14. At the same time the number of possible paths increases from 120 to 18,651,552,840. For hypercubes of dimension $L \geq 6$ the total number $P_{L}$ of self-avoiding paths connecting two antipodal corners is not explicitly known, but it can be shown to grow double-exponentially as [6]

$$
\lim _{L \rightarrow \infty} \frac{\ln \ln P_{L}}{L}=\ln 2 .
$$

This coincides with the behaviour of the naive estimate $P_{L} \sim L^{2^{L}}$ obtained by noting that a self-avoiding path takes at most $2^{L}$ steps, and that each step can proceed in $L$ different directions.

In the following we consider the binary hypercube of dimension $L$ and ask for the number of general (undirected) accessible paths connecting two genotypes $x, y$ at distance $D=d(x, y)$ that differ in fitness by $\beta=f_{y}-f_{x}>0$. An expression for the expected number of paths can be formally written down along the lines of Eq. (5) as

$$
\mathbb{E}_{L, D, \beta}\left(X_{x, y}\right)=\sum_{k \geq 0} a_{L, D, k} \frac{\beta^{D+2 k-1}}{(D+2 k-1) !},
$$

where $a_{L, D, k}$ is the number of self-avoiding paths with $k$ reversions that connect two genotypes at distance $D$ on a hypercube of dimension $L$. Through a careful analysis of the asymptotics of the $a_{L, D, k}$, Berestycki et al. showed that in the joint limit $L, D \rightarrow \infty$ at fixed $\alpha=D / L$ the exponential growth rate of the expected number of accessible paths is given by [6]

$$
\lim _{L \rightarrow \infty}\left[\mathbb{E}_{L, \alpha L, \beta}\left(X_{x, y}\right)\right]^{1 / L}=\sinh (\beta)^{\alpha} \cosh (\beta)^{1-\alpha} .
$$

Similar to the directed path case discussed previously, when the right hand side of (11) is less than 1, Markov's inequality implies $\lim _{L \rightarrow \infty} \mathbb{P}_{L, \alpha L, \beta}\left(X_{x, y} \geq 1\right)=0$. The condition $\sinh \left(\beta^{*}\right)^{\alpha} \cosh \left(\beta^{*}\right)^{1-\alpha}=1$ defines a function $\beta^{*}(\alpha)$ that takes on its maximal value $\beta^{*}(1)=\ln (1+\sqrt{2}) \approx 0.88137 \ldots$ at $\alpha=1$ and tends to 0 for $\alpha \rightarrow 0$. Berestycki et al. conjectured that, similar to the directed case, the expectation (11) "tells the truth", in the sense that $\lim _{L \rightarrow \infty} \mathbb{P}_{L, \alpha L, \beta}\left(X_{x, y} \geq 1\right)=1$ when $\beta>\beta^{*}(\alpha)$. This conjecture was proven independently by Martinsson [40] and Li [36]. Martinsson's proofis makes use of an ingenious mapping to first passage percolation on the hypercube, which allows him to refer to earlier results for the latter problem [41].

Thus we see that the extension to undirected paths fundamentally changes the nature of the problem, in that the transition to high accessibility now occurs at a nontrivial threshold fitness $\beta^{*}<1$. Moreover, the fact that $\beta^{*}$ decreases with decreasing $\alpha$ shows that, in contrast to the directed path case, the genotypes that do not lie "between" the initial and final point of the path $\amalg$ cannot be ignored. Evolutionary accessibility increases when $D$ decreases relative to $L$ because of the contribution from

$\S$ For technical reasons Martinsson's proof is limited to the range $\alpha \geq 0.002$.

II In biological terms, these are genotypes that cannot be generated from the initial and final genotype by crossover. 
paths that accumulate and later revert mutations that are part of neither the initial nor the final genotype.

\subsection{Multiple alleles}

In sequence spaces with more than two alleles $(a>2)$ mutational paths can include "sideways" steps where the distance to the initial and final point neither decreases nor increases, because a site mutates to an allele that is contained in neither the initial nor the final genotype. Zagorski et al. carried out simulations of mutational paths in multiallelic sequence spaces and found a significant increase of accessibility with increasing $a$ that is caused mainly by sideways steps [69].

In the following we summarise the main results of a recent analytic study of this problem [61]. For this purpose we formalise the mutational structure on the allele set $\mathcal{A}$ through the adjacency matrix $\mathbf{A}$ of the mutation graph, with elements $A_{k k}=0$ and $A_{k l}=1$ iff mutations can occur from allele $k$ to allele $l$, where $k, l \in \mathcal{A}$. Then the exponential growth rate of the expected number of accessible paths between two genotypes $x, y$ is given by

$$
\lim _{L \rightarrow \infty}\left[\mathbb{E}_{L, \mathbf{A}, \beta}\left(X_{x, y}\right)\right]^{1 / L}=\prod_{k, l=1}^{a}\left[\left(e^{\beta \mathbf{A}}\right)_{k l}\right]^{p_{k l}},
$$

where $p_{k l}$ denotes the fraction of sites at which $\tau_{i}(x)=k$ and $\tau_{i}(y)=l$ in the joint limit

$d(x, y) \rightarrow \infty$ and $L \rightarrow \infty$. The information about the Hamming distance between $x$ and $y$ is contained in the $p_{k l}$ through the relation

$$
\lim _{L \rightarrow \infty} \frac{d(x, y)}{L}=1-\sum_{k=1}^{a} p_{k k},
$$

but in general the number of paths depends on the entire allelic composition of the initial and final genotypes. An important special case is the complete mutation graph, where $A_{k l}=1-\delta_{k l}$. In the biallelic case considered previously this implies $\mathbf{A}^{2}=\mathbf{1}$, and therefore $e^{\beta \mathbf{A}}=\sinh (\beta) \mathbf{A}+\cosh (\beta) \mathbf{1}$. Observing further that $p_{01}+p_{10}=\alpha$ and $p_{11}+p_{00}=1-\alpha$, the expression (12) is seen to reduce to (11).

As before, the expression (12) can be invoked together with Markov's inequality to derive a lower bound $\beta^{*}$ on the critical fitness difference below which $\lim _{L \rightarrow \infty} \mathbb{P}_{L, \mathbf{A}, \beta}\left(X_{x, y} \geq 1\right)=0$. For the complete mutation graph over $a$ alleles and initial and final genotypes at maximal distance $d=L$, the equation for $\beta^{*}(a)$ reads

$$
\frac{1}{a}\left(e^{(a-1) \beta^{*}}-e^{-\beta^{*}}\right)=1,
$$

which can be solved explicitly for $a \leq 4$. In particular, for the case of the 4-letter nucleotide alphabets of RNA and DNA the threshold fitness is

$$
\beta^{*}(4)=\ln \left(\frac{1}{\sqrt{2}}+\sqrt{\left(\sqrt{2}-\frac{1}{2}\right)}\right) \approx 0.5088 \ldots
$$


For large $a$ the solution of (14) can be approximated as

$$
\beta^{*}(a)=\frac{\ln (a)}{a}+\frac{1+\ln (a)}{a^{2}}+\mathcal{O}\left(\frac{\ln (a)}{a^{3}}\right) .
$$

For the 20 -letter amino acid alphabet this yields the estimate $\beta^{*} \approx 0.1598 \ldots$. Because of the restrictions of the genetic code, the amino acid mutation graph is not complete, but a calculation based on the actual mutation graph shows that this only leads to minor deviations from this estimate.

The comparison to numerical simulations [69] and related results for first passage percolation [42] indicate that the bound $\beta^{*}$ given by setting the right hand side of (12) to unity is tight at least for the complete graph, and presumably also for a rather general class of allelic mutation graphs. An interesting application for which the lower bound provided by (12) suffices is the linear graph $\mathbb{q}$, where allele $k$ is allowed to mutate only to the neighbouring alleles $k \pm 1$ for $1 \leq k \leq a-2$, and the boundary alleles mutate as $0 \rightarrow 1$ and $a-1 \rightarrow a-2$. For paths connecting the boundary genotypes $x, y$ with $\tau_{i}(x)=0$ and $\tau_{i}(y)=a-1$ or vice versa, one finds that $\beta^{*}>1$ for $a>2$, which implies that accessible paths do not exist for any value of $\beta \in[0,1]$.

\section{Accessibility percolation on trees}

Computing higher moments of $X_{x, y}$ on the directed hypercube is difficult because different paths can merge and diverge multiple times [26]. The observation that sequence spaces are nevertheless essentially tree-like for large $L$ has motivated a number of studies of accessibility percolation on trees, where this problem does not arise.

We begin with a regular rooted $n$-tree of height $h$ [46]. The tree has $n^{h}$ leaves and equally many paths of length $h+1$ from the root to one of the leaves. The nodes and leaves are labelled by continuous, independent and identically distributed (i.i.d.) random numbers, and therefore the probability for a given path to be accessible is $1 /(h+1)$ !. Since the exponential growth of the number of paths cannot compensate the factorial decrease in probability, the usual first moment bound shows that accessible paths do not exist for $h \rightarrow \infty$ for any fixed value of $n$. One is thus lead to consider trees where the branching number grows with increasing height according to a function $n(h)$. The expected number of accessible paths is then $\dagger$

$$
\mathbb{E}_{h, n(h)}(X)=\frac{n(h)^{h}}{(h+1) !} \sim \frac{[e n(h) / h]^{h}}{\sqrt{2 \pi h}}
$$

for large $h$, which suggests that the transition to high accessibility occurs for linear functions $n(h)=\lambda h$ with $\lambda>\lambda^{*} \geq 1 / e$. In [46] the upper bound $\lambda^{*} \leq 1$ was obtained using the second moment inequality ([6), and a subsequent refined analysis showed that the lower bound is tight and $\lambda^{*}=1 / e$ exactly [58]. The linear growth $n \sim h$ corresponds

I A possible biological interpretation of the linear mutation graph is that alleles represent copy-number variants of genes [2]. In this case the assignment of random fitness values is however not very plausible. $\dagger$ Throughout this section paths are assumed to go from the root to a leaf of the tree. The indices $x, y$ of $X$ indicating the start and end point of the paths are therefore omitted. 
to the geometry of the directed hypercubet, which can be viewed as a directed graph whose vertex degree and diameter are both equal to $L$. The fact that trees with linear growth are marginally accessible is thus consistent with the results for the directed hypercube described in Sec. 2.1,

A similar conclusion can be drawn from a subtly different analysis carried out by Coletti et al. [11, who consider infinite trees with the branching number at level $l$ given by an increasing function $n(l)$ (the root is located at $l=0$ ). For a linear growth function $n(l)=l+1$ the number of leaves (and hence the number of distinct paths from the root) at height $h$ is $h$ !, the same as the number of directed paths on a hypercube of dimension $h$. Without constraints on the fitness of the root the probability for a path to be accessible is again $1 /(h+1)$ !. The expected number of paths is

$$
\mathbb{E}_{h, n(l)=l+1}(X)=\frac{h !}{(h+1) !}=\frac{1}{h+1}
$$

and there is no accessibility for $h \rightarrow \infty$. The main result established in [11] is that this case is marginal in the sense that the probability for existence of accessible paths is positive for growth functions $n(l)=\left\lceil(l+1)^{\gamma}\right\rceil$ with $\gamma>1$.

Instead of letting the branching number of the tree grow with its height, accessibility can also be increased by introducing a bias on the random fitness variables [46]. Specifically, we take the fitness of a node $x$ at distance $l$ from the root to be of the form

$$
f_{x}=\xi_{x}+c l
$$

where $c>0$ and the $\xi_{x}$ are continuous i.i.d. random variables. The linear trend in (19) increases the likelihood of the variables to be in increasing order in a way that depends on the distribution of the $\xi_{x}$. For the case of the Gumbel distribution $\mathbb{P}\left(\xi_{x}<z\right)=\exp \left[-e^{-z}\right]$, the ordering probability $\mathbb{P}\left(f_{x_{1}}<f_{x_{2}}<\cdots<f_{x_{h}}\right)$, which is also the probability for a path of length $h$ to be accessible, can be shown to be given by [21]

$$
\mathbb{P}\left(f_{x_{1}}<f_{x_{2}}<\cdots<f_{x_{h}}\right)=\frac{\left(1-e^{-c}\right)^{h}}{\prod_{l=1}^{h}\left(1-e^{-c l}\right)}=\frac{1}{[h]_{e^{-c}} !} .
$$

Here

$$
[k]_{q} ! \equiv \prod_{j=1}^{k}[j]_{q}, \quad[j]_{q} \equiv \frac{1-q^{j}}{1-q}, \quad q \in[0,1],
$$

defines the $q$-factorial of a $q$-number $\xi[k]_{q}[34$. Note that for $q \rightarrow 1$ the standard factorial is retrieved. The result (20) was first derived in the context of record statistics, where it describes the probability for all entries in a sequence of random variables with a linear trend to be records $\llbracket$ [21].

Since the product in the denominator of (20) converges for $h \rightarrow \infty$, for a tree of fixed branching number $n$, the expected number of accessible paths grows or shrinks

† See [5] for a precise way of approximating the directed hypercube by a tree.

$\S$ For a related application of $q$-factorials see 52 .

I| A similar relation between record statistics and accessibility was described in [11. 
exponentially as $\left[n\left(1-e^{-c}\right)\right]^{h}$ for large $h$. This suggests that the accessibility transition occurs when $n\left(1-e^{-c}\right)=1$ or $c=c^{*}$ with

$$
c^{*}=\ln \left(\frac{n}{n-1}\right) \text {. }
$$

An analysis using the second moment inequality (6) confirms this expectation and moreover provides the lower bound

$$
\lim _{h \rightarrow \infty} \mathbb{P}_{h, n, c}(X \geq 1) \geq \sqrt{\frac{2 \pi}{c}} \exp \left(\frac{c}{24}-\frac{\pi}{6 c}\right)\left[e^{-c^{*}}-e^{-c}\right]
$$

for $c>c^{*}$ [46].

\section{Correlated fitness landscapes}

Although the assumption of i.i.d. random fitness values is mathematically convenient, it cannot be expected to be biologically realistic. Indeed, analyses of experimental data generally show that real fitness landscapes are less rugged than predicted by the i.i.d. model [4, 16, 22, 64. For this reason several classes of probabilistic fitness landscapes with tunable fitness correlations have been proposed. In contrast to the i.i.d. case, in these models the rank ordering of genotypes (and hence the accessibility of mutational paths) generally depends on the base distribution of the random variables from which the landscape is constructed. In the following we focus on properties for which this dependence does not matter, and consider biallelic sequence spaces $(a=2)$ throughout.

In the rough Mount Fuji (RMF) model, i.i.d. random fitness values are combined additively with a linear $\square$ function of the distance to a reference genotype $x_{0}$, such that

$$
f_{x}=\xi_{x}+c d\left(x, x_{0}\right)
$$

with $c>0$ and continuous i.i.d. random variables $\xi_{x}$ [45]. This is obviously very similar to the fitness assignment (19) in the tree model with bias discussed in Sec. 3. The RMF model on the directed hypercube was analysed by Hegarty and Martinsson [26], who showed that accessible paths of length $L$ starting at $x_{0}$ exist with probability converging to 1 for any $c>0$ and $L \rightarrow \infty$. The effect of the bias in the undirected case is less clear cut, since mutational reversions are discouraged when $c>0$. In particular, for $c \rightarrow \infty$ all directed paths become accessible and no back steps can occur. Numerical simulations for small systems suggest that the competition between directed and undirected paths leads to a maximum in the number of accessible paths at an intermediate value of $c$ [30].

The NK-model $\oplus$ originally introduced by Kauffman and Weinberger [32, 33. constitute a popular and versatile class of correlated fitness landscapes that continues to attract the attention of diverse research communities (see [27] for a recent review). The basic structural element of the model are the interaction sets $B_{i} \subset \mathcal{L}$ of genetic loci, which are subsets of the locus set $\mathcal{L}=\{1,2, \cdots, L\}$. In the most commonly used setting

9 For a generalisation to nonlinear functions see [51, 53.

+ The acronym refers to the number of loci $N$ (here denoted by $L$ ) and the number of interaction partners $K$ of a locus (here denoted by $k-1$ ). 

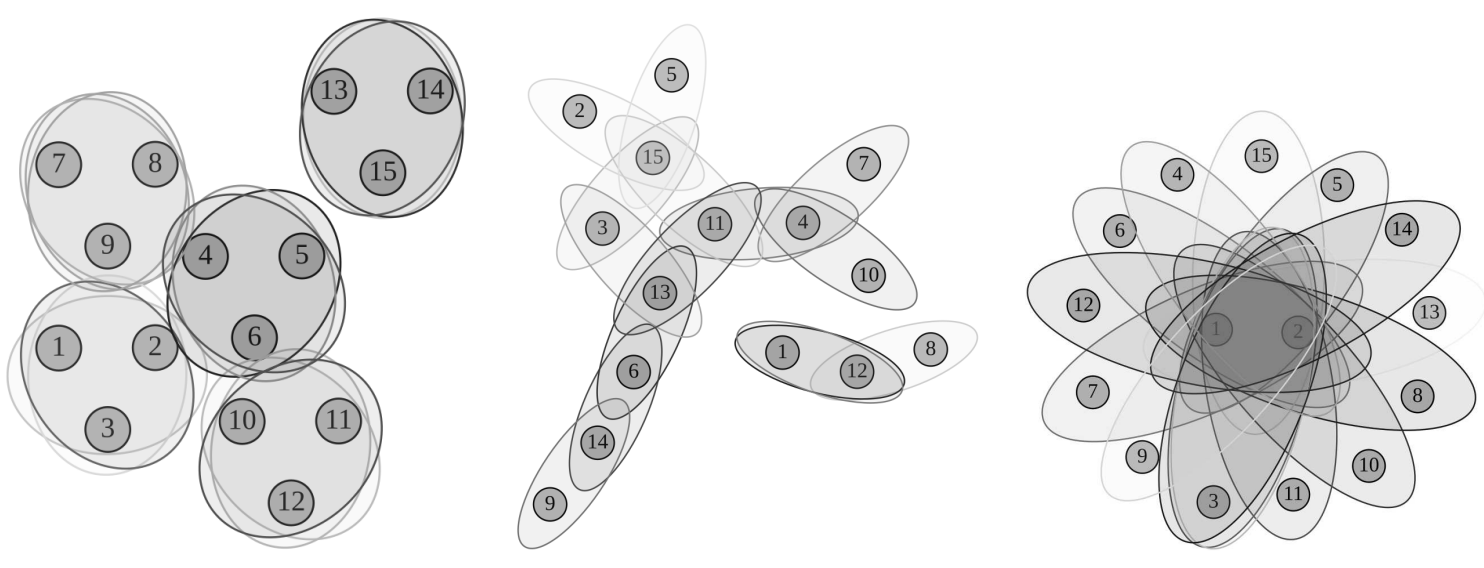

Figure 2. Three examples of NK interaction structures for $L=15$ loci. Interaction sets are shown as ellipses. Left panel: Block structure [50, 54] with $k=3$. The interaction sets are disjoint and form five triples of identical sets. Middle panel: Random structure with $k=2$. Loci are assigned randomly to interaction sets subject to the constraints described in the text. Right panel: Star structure with $k=3$. Loci 1 and 2 are center loci that are contained in all interaction sets. The interaction sets associated with the remaining 13 ray loci contain only the locus itself and the center loci (modified from [27]).

there are $L$ interaction sets of equal size $\left|B_{i}\right|=k, 1 \leq k \leq L$, and moreover it is assumed that locus $i$ belongs to its own interaction set, $i \in B_{i}$. Loci within one interaction set affect each others fitness effects in a random manner, whereas the fitness effects from different interaction sets combine additively. This is implemented by writing the fitness of a genotype $x$ as a sum over contributions from the interaction sets,

$$
f_{x}=\sum_{i=1}^{L} \phi_{k}^{(i)}\left(\downarrow_{B_{i}} x\right)
$$

Here the $\phi_{k}^{(i)}$ are functions on the $k$-dimensional hypercube which assign a continuous i.i.d. random number to each of the $2^{k}$ sequences in $\mathbb{H}_{2}^{k}$, and $\downarrow_{B_{i}}: \mathbb{H}_{2}^{L} \rightarrow \mathbb{H}_{2}^{k}$ projects the genotype sequence onto the interaction set according to

$$
\tau_{i}\left(\downarrow_{\mathcal{S}} x\right)=\tau_{i}(x) \forall i \in \mathcal{S} \subset \mathcal{L} .
$$

The functions $\phi_{k}^{(i)}$ for different $i$ are independent. The choice of the interaction sets defines the interaction structure of the model [27, 47], see Fig. 2 for illustration.

The correlations in the fitness landscape are tuned through the size $k$ of the interaction sets [9]. For $k=L$ every interaction set contains all loci and the model reduces to the uncorrelated landscape. On the other hand, when $k=1$ the fitness effects of different loci combine additively according to

$$
f_{x}=\sum_{i=1}^{L} \phi_{1}^{(i)}(0)+\sum_{i=1}^{L}\left(\phi_{1}^{(i)}(1)-\phi_{1}^{(i)}(0)\right) \tau_{i}(x),
$$

where the $\phi_{1}^{(i)}(0)$ and $\phi_{1}^{(i)}(1)$ are continuous i.i.d. random variables. The additive 
landscape has a unique maximum and all directed paths to the maximum are accessible [67].

For general $k$, the analysis of directed accessible paths is relatively straightforward for the block structure shown in the left panel of Fig. 2 [59]. Note first that in this case the $L$ interaction sets fall into $b=L / k$ groups* within which the sets are identical, and therefore only $b$ interaction sets have to be distinguished. Following the setting originally introduced in [22, we consider directed paths of length $L$ that end at the global maximum $x_{\max }$ of the fitness landscape and start at its antipodal point $\bar{x}_{\max }$ defined by $\tau_{i}\left(\bar{x}_{\max }\right)=1-\tau_{i}\left(x_{\max }\right)$. Along such a path each locus has to be mutated once. Since the interaction sets are disjoint, each mutation event occurs in one of the sets and changes only the fitness contribution corresponding to this set (compare to (25)). In this way the path can be decomposed into $b$ sub-paths of length $k$ on $\mathbb{H}_{2}^{k}$, each of which remains within one interaction set. The global path is accessible iff all the sub-paths are. Denoting the number of accessible paths in the interaction set $B_{i}$ by $X_{k}^{(i)}$, the number of global paths is thus

$$
X_{L, k}^{\text {block }}=\frac{L !}{(k !)^{b}} \prod_{i=1}^{b} X_{k}^{(i)} .
$$

The combinatorial prefactor describes the number of ways in which a given set of subpaths can be combined into a global path. An immediate consequence is that $X_{L, k}^{\text {block }}$ is a non-negative multiple of $\frac{L !}{(k !)^{b}}$.

Since the fitness contributions within each interaction set are continuous i.i.d. random variables, the statistics of the $X_{k}^{(i)}$ are given by the uncorrelated model discussed in Sect. 2.1. In particular, it follows from (8) that $\mathbb{E}\left(X_{k}^{(i)}\right)=1$ independent of $k$, and therefore

$$
\mathbb{E}\left(X_{L, k}^{\text {block }}\right)=\frac{L !}{(k !)^{b}}
$$

Similarly,

$$
\mathbb{P}\left(X_{L, k}^{\text {block }} \geq 1\right)=\left[\mathbb{P}\left(X_{k} \geq 1\right)\right]^{b},
$$

where $X_{k}$ is the number of accessible directed paths in the uncorrelated model on $\mathbb{H}_{2}^{k}$ that end at the global maximum and start at the (unconstrained) antipodal point. The results described in Sec. 2.1 imply that $\mathbb{P}\left(X_{k} \geq 1\right)<1$ for any $k \geq 2$, and we conclude that

$$
\lim _{L \rightarrow \infty} \mathbb{P}\left(X_{L, k}^{\text {block }} \geq 1\right)=0
$$

for any fixed $k \geq 2$. Taken together, the results (29) and (31) show that the accessibility properties of the NK model with block interactions are strikingly different from those of the uncorrelated model, in that the expected number of accessible paths grows factorially with $L$, while at the same time the probability that the landscape is accessible

* We assume here that $L$ is divisible by $k$.

$\sharp$ Specifically, $\mathbb{P}\left(X_{2} \geq 1\right)=2 / 3$ and $\mathbb{P}\left(X_{3} \geq 1\right)=97 / 210 \approx 0.462 \ldots$ [59]. 
vanishes exponentially. It is clear from the second moment inequality (6) that these two statements are compatible only if the coefficient of variation of $X_{L, k}^{\text {block }}$ diverges with $L$. This is indeed the case and follows from the multiplicative structure of (28) [59].

An early numerical investigation indicated that the accessibility of NK landscapes depends on the interaction structure in a significant and complicated way [23, but subsequent work has shown that the behaviour of the block structure described above is quite representative at least asymptotically for large $L$. In brief, it has been proved that for a large class of interaction structures characterised as locally bounded, the probability $\mathbb{P}\left(X_{L, k} \geq 1\right)$ vanishes exponentially in $L$ for $L \rightarrow \infty$ and any fixed $k \geq 2$ [27, 60]. The proof relies on the inevitable existence of a certain local genetic interaction motif known as reciprocal sign epistasis [55] which prevents an accessible path (directed or undirected) from traversing the hypercube and hence decomposes the genotype space into mutually inaccessible domains. Most commonly studied interaction structures, including the random structure depicted in the middle panel of Fig. 2 are locally bounded, but the star structure shown in the right panel of the figure is not.

\section{Paths with valley crossing}

In this section we consider the consequences of relaxing the condition of strict monotonicity on accessible paths. Theoretical studies of populations that cross a fitness valley have shown that different dynamic modes for this process have to be distinguished [66]. In small population $\uplus$ a deleterious mutation that decreases fitness can fix, which implies that the entire population moves to a state of lower fitness and continues its trajectory from there. By contrast, in larger populations a small sub-population of valley genotypes is maintained by mutation-selection balance, and if a target genotype that is fitter than both the initial and the valley genotypes exists, it can arise by mutation from the valley population. In the latter case the majority of the population never resides in the valley, and for this reason the process is also known as stochastic tunnelling [28]. Increasing population size even further, eventually double mutations become likely. In the following we focus on the regime where multiple mutations can be neglected and valleys are crossed either by fixation or stochastic tunnelling.

These two modes of valley crossing impose different conditions on the fitness values along the evolutionary trajectory [13]. For the fixation mode the crossing event implies an unconditional decreases of the fitness values, after which the monotonic increase in fitness is resumed until the next valley crossing. By contrast, when valley crossing occurs in the (stochastic) tunnelling mode, the mutational step out of the valley has to overcompensate the fitness decrease of the preceding step. Denoting the valley genotype by $v$, the fitness values along the sub-trajectory $x \rightarrow v \rightarrow x^{\prime}$ have to satisfy the condition $f_{x^{\prime}}>f_{x}>f_{v}$, see Fig. 3 for illustration. In the following we examine the effect of allowing for one or several valley crossings on the accessibility of directed paths of $\dagger$ The condition on the population size $N$ is $N \Delta f \sim 1$, where $\Delta f=f_{x}-f_{v}>0$ is the fitness difference between the initial genotype $x$ and the valley genotype $v$. 


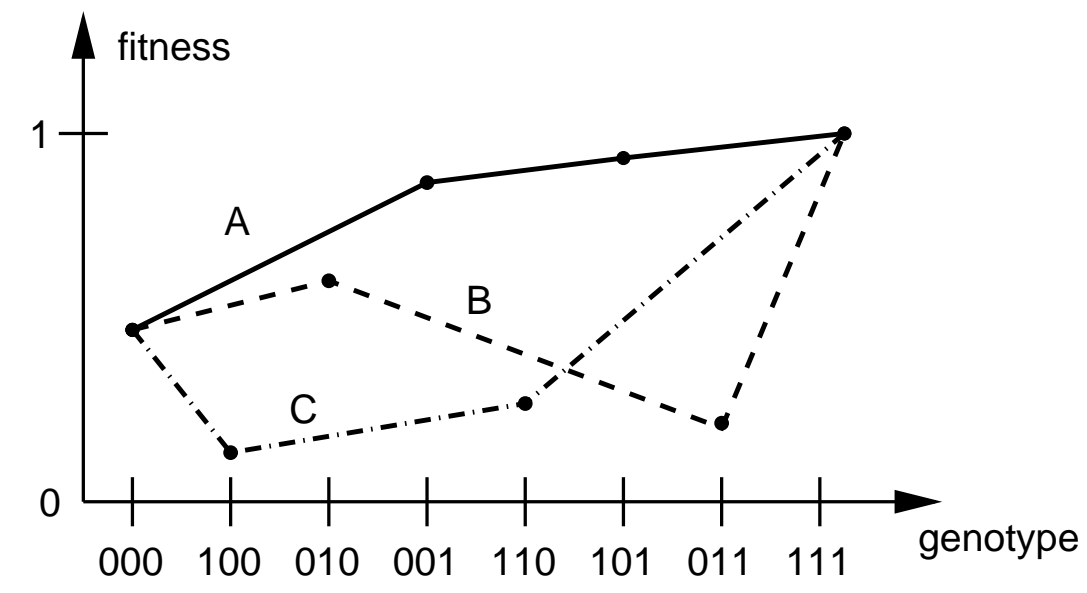

Figure 3. Three directed paths in a three-dimensional hypercube that illustrate the different modes of valley crossing. Path A (full) is monotonic, path B (dashed) contains a stochastic tunnelling event in the second step, and along path $\mathrm{C}$ (dash-dotted) a deleterious mutation fixes in the first step.

length $\ell$ on the hypercube. Fitness values are drawn independently from the uniform distribution on $[0,1]$, and the final genotype $y$ is assumed to have maximal fitness, $f_{y}=1$.

We first show that a single valley crossing of the fixation type suffices to induce high accessibility. Starting at an arbitrary initial genotype $x$ with fitness $f_{x}$, we make use of the crossing event in the first step to move to the genotype $x_{1}$ that has the lowest fitness among the $\ell$ available neighbours. The probability distribution function of this fitness value is given by

$$
\mathbb{P}\left(f_{x_{1}}<z\right)=1-(1-z)^{\ell} \rightarrow 1-e^{-z \ell}
$$

for large $\ell$ and small $z$, and using (7) we conclude that an accessible path will be found with certainty from the second step onward. The expected number of accessible paths starting from a random fitness value can be shown to grow exponentially as $2^{\ell}-\ell$ in this setting [13].

The analysis of paths with stochastic tunnelling events is more subtle $₫$. Following the setup of Sect. 2.1, we fix the initial and final fitness values at $f_{x}=1-\beta$ and $f_{y}=1$, respectively. We consider paths of length $\ell$ with a fixed number $t$ of tunnelling events, and denote by $\psi_{t}(\ell, \beta)$ the probability that such a path is accessible. We know from (5) that $\psi_{0}(\ell, \beta)=\frac{\beta^{\ell-1}}{(\ell-1) !}$. For general $t$ and $\ell \geq 3$, the $\psi_{t}$ satisfy the recursion relation

$$
\psi_{t}(\ell, \beta)=(1-\beta) \int_{1-\beta}^{1} d u \psi_{t-1}(\ell-2, u)+\int_{1-\beta}^{1} d u \psi_{t}(\ell-1, u) .
$$

The first term on the right hand side describes the situation when the first step is a tunnelling event. This implies that the second fitness value $f_{x_{1}} \in[0,1-\beta]$, which happens with probability $1-\beta$, whereas the subsequent value $u=f_{x_{2}}$ has to be in ‡ This part is based on unpublished notes by Éric Brunet [8]. 
$[1-\beta, 1]$. From this point on one has to cover the remaining $\ell-2$ steps using $t-1$ tunnelling events. The second term covers the cases where fitness increases in the first step, such that $f_{x_{1}}=u \in[1-\beta, 1]$ and the remaining $\ell-1$ steps can make use of all $t$ tunnelling events. The solution of (33) with the appropriate boundary conditions is given by

$$
\begin{aligned}
\psi_{t}(\ell, \beta) & =\frac{1}{2^{t} t !(\ell-2 t) !} \beta^{\ell-t}(2-\beta)^{t}\left[\frac{\ell-t}{\beta}-\frac{t}{2-\beta}\right] \\
& =\frac{1}{2^{t} t !(\ell-2 t) !} \frac{d}{d \beta}\left[\beta^{\ell-t}(2-\beta)^{t}\right] .
\end{aligned}
$$

Integrating (34) with respect to $\beta$ and multiplying by the total number of directed paths $\ell$ ! one obtains the expected number of paths with arbitrary starting fitness as

$$
\mathbb{E}\left(X_{\ell, t}\right)=\frac{\ell !}{2^{t} t !(\ell-2 t) !} \propto \ell^{2 t}
$$

for large $\ell$ and fixed $t$, which generalises (8) to $t \geq 1$. Although this suggests a significant increase in accessibility, the algebraic growth in $\ell$ is not sufficient to overcome the exponential reduction in probability caused by the factor $\beta^{\ell-t}$ in (34). Conditioned on the initial fitness, the expected number of paths behaves as

$$
\mathbb{E}\left(X_{\ell, t, \beta}\right) \propto \frac{\ell^{2 t+1}}{2^{t} t !} \beta^{\ell-t-1}(2-\beta)^{t} \propto \ell^{2 t+1} \beta^{\ell}
$$

for large $\ell$ and fixed $t$, which converges to zero for any fixed $\beta<1$. Setting $\beta=\beta_{\ell}=1-\epsilon_{\ell}$ with $\lim _{\ell \rightarrow \infty} \epsilon_{\ell}=0$, we see that a necessary condition for accessibility is $\epsilon_{\ell}<(2 t+1) \frac{\ln \ell}{\ell}$, which is only a small improvement compared to the case without valley crossings in (7). We conclude that the effects of the two modes of valley crossing on accessibility are very different.

Duque et al. recently studied accessibility percolation with valley crossings on $n$ trees with a height-dependent branching number $n(h)$, compare to Sec. 3. They define a path to be $k$-accessible if any $k$ consecutive fitness values along the path contain at least one element of a monotonically increasing sub-sequence, and show that the critical growth of $n(h)$ required to guarantee accessibility is given by $n(h) \propto[h /(e k)]^{1 / k}[18$.

\section{Summary and conclusions}

\subsection{Accessibility and predictability}

The results reviewed in the preceding sections reveal a variety of mathematical mechanisms by which evolutionary accessibility can arise in high-dimensional genotype spaces. The directed hypercube with continuous i.i.d. fitness values [10, 22] turns out to be marginally accessible, in the sense that accessible paths exist only if the fitness difference between the initial and final genotype is as large as possible [26]. Allowing for undirected paths lowers the threshold for accessibility to a nontrivial value $\beta^{*} \in(0,1)$ [6, 36, 40]. In multiallelic sequence spaces $\beta^{*}$ depends on the graph of allowed mutational transitions on the set of alleles $\mathcal{A}$. If the mutation graph is complete, $\beta^{*}$ decreases with 
increasing number of alleles and tends to zero for $a \rightarrow \infty$ [61, 69]. When the genotype space is a tree, accessibility percolation occurs as a function of tree geometry [11, 46, 58, or as a function of a bias imposed on the fitness values [46]. Apart from the last example, in all cases the transition is characterised by a discontinuous change of the limiting value of $\mathbb{P}(X \geq 1)$ from 0 to 1 .

On correlated fitness landscapes of NK-type, accessibility is determined by the graph of interactions among loci, and accessible paths do not exist for interaction structures that are locally bounded [27, 60]. This general result as well as the results for the explicitly solvable block model [59] show that, despite being less rugged (more correlated) than the uncorrelated model, NK-landscapes are much less accessible. Evolutionary accessibility is thus not strictly linked to other measures of fitness landscape ruggedness such as the number of local fitness maxima [64].

The concept of accessible paths was originally introduced in the context of evolutionary predictability. Following the interpretation that Weinreich et al. applied to their seminal experiment on antibiotic resistance evolution [68], the evolutionary trajectories connecting an initial to a final genotype are highly predictable if a small but nonzero number of accessible pathways exist. Referring to the results described in Sect. 2, we see that this condition is approximately satisfied for the directed hypercube with $\beta=1$, where $\lim _{L \rightarrow \infty} \mathbb{P}(X \geq 1)=1$ and the expected number of paths is equal to $L$ and hence much smaller than the total number of paths $L$ !. For the undirected hypercube at $\beta>\beta^{*}$ the number of accessible paths increases exponentially in $L$ but is still much smaller than the total number of paths. In this sense the uncorrelated model can be said to conform to the scenario of high predictability envisioned in [68]. However, the results for the NK-models discussed in Sect. 4 show that other scenarios are possible as well. According to Eqs (29), in the NK-model with block interactions accessible paths typically do not exist, but if they do their number grows factorially in $L$, leading to low predictability.

\subsection{Accessible paths and evolutionary dynamics}

Formalising evolutionary accessibility through fitness-monotonic mutational paths is conceptually appealing, because it does not require any assumptions about the evolutionary dynamics. On the other hand, this simplification also limits the applicability of the theory to actual evolutionary processes. The paradigm of evolutionary dynamics underlying the notion of accessible paths, known as the strong selection-weak mutation (SSWM) regime, applies in a window of population size $N$ where mutations are rares, $N \mu \ll 1$, and selection is strong, $N|\Delta f| \gg 1$ [25, 48]. Under these conditions the population is almost always monomorphic and can evolve only by fixing beneficial mutations, which implies that it is constrained to move along accessible paths. A rigorous derivation of the SSWM limit from microscopic adaptive dynamics

$\S$ Here $\mu$ denotes the mutation rate per individual and generation, and $\Delta f$ is the typical fitness difference between neighbouring genotypes. 
is described in the chapter by Anton Bovier [7. The SSWM framework allows one to assign a weight to each accessible path, which is given by the product of the relative fixation probabilities along the path. Empirical studies indicate that these weights are often strongly concentrated on a small number of paths [38, 68]. As a consequence the evolutionary predictability may be even higher than expected based only on the number of accessible paths.

However, evolving populations are myopic and lack the foresight required to determine which of the many available paths will eventually lead to high fitness. Studies of SSWM dynamics on the $L$-dimensional hypercube with uncorrelated fitness values have shown that adaptive walks governed by local dynamics typically terminate at a local fitness peak after $\sim \ln L$ steps and are thus much shorter than the accessible paths considered in this chapter $[19,39,44,48]$. Greedy adaptive walks that always fix the most beneficial mutation terminate already after $e-1 \approx 1.718$ steps [49]. Walks following a (biologically unrealistic) 'reluctant' dynamics by always choosing the smallest available positive fitness difference take $\mathcal{O}(L)$ steps but do not reach fitness levels comparable to the globally maximal fitness [47]. The behaviour of adaptive walks on correlated fitness landscapes is more complex, but studies of the RMF model have shown that the available, long accessible paths are dynamically relevant only if the bias parameter $c$ in (24) is sufficiently large and/or the distribution of the random component $\xi_{x}$ is sufficiently heavy tailed [51, 52, 53]. Typical walk lengths in the NK-model at fixed $k$ are of the order of (but smaller than) $L$ [47].

When the population size increases beyond the weak mutation regime additional complications arise. On the one hand, the simultaneous presence of multiple mutation clones in the population implies an advantage for mutations of large beneficial effect, such that the dynamics becomes increasingly greedy and hence deterministic [29, 52]. On the other hand, a higher mutation rate facilitates the crossing of fitness valleys, which strongly increases the number of available paths. A detailed numerical study has shown that the distinguished role of fitness-monotonic paths is largely lost in this regime, and moreover the interplay of the two effects mentioned above leads to a non-monotonic dependence of predictability on population size [65].

Taken together, the considerations sketched in this section make it clear that the investigation of accessible paths only constitutes a first step towards a broader understanding of evolutionary predictability.

Acknowledgement. The work described here is the joint effort of a large number of collaborators. I am particularly grateful to Éric Brunet, Lucas Deecke, Jasper Franke, Mario Josupeit, Alexander Klözer, Stefan Nowak and Benjamin Schmiegelt for their contributions to this project, and to Benjamin Schmiegelt for a critical reading of the manuscript.

[1] N. Alon and J. Spencer, The Probabilistic Method, 2nd ed., Wiley, New York, 2000.

[2] L. Altenberg, Fundamental properties of the evolution of mutational robustness, arXiv:1508.07866 (2015). 
[3] F. H. Arnold, The library of Maynard-Smith: My search for meaning in the protein universe, Microbe 6 (2011), 316-318.

[4] C. Bank, S. Matuszewski, R. T. Hietpas and J. D. Jensen, On the (un)predictability of a large intragenic fitness landscape, Proc. Natl. Acad. Sci. USA 113 (2016), 14085-14090.

[5] J. Berestycki, É. Brunet and Z. Shi, The number of accessible paths in the hypercube, Bernoulli 22 (2016), 653-680.

[6] J. Berestycki, É. Brunet and Z. Shi, Accessibility percolation with backsteps, ALEA, Lat. Am. J. Probab. Math. Stat. 14 (2017), 45-62.

[7] A. Bovier, Stochastic models for adaptive dynamics: Scaling limits and diversity. In: Probabilistic Structures in Evolution (eds. E. Baake and A. Wakolbinger), EMS Press, Berlin (2021), 127-149.

[8] É. Brunet, private communication (2015).

[9] P. R. A. Campos, C. Adami and C. O. Wilke, Optimal adaptive performance and delocalization in NK fitness landscapes, Physica A 304 (2002), 495-506; erratum ibid. 318 (2003), 637.

[10] M. Carneiro and D. L. Hartl, Adaptive landscapes and protein evolution, Proc. Natl. Acad. Sci. USA 107, 1747-1751.

[11] C. F. Coletti, R. J. Gava and P. M. Rodríguez, On the existence of accessibility in a tree-indexed percolation model, Physica A 492 (2018), 382-388.

[12] K. Crona, D. Green and M. Barlow, The peaks and geometry of fitness landscapes, J. Theor. Biol. 317 (2013), 1-10.

[13] L. A. C. Deecke, Fitness landscapes and evolutionary accessibility: The effect of downhill steps, Bachelor thesis, University of Cologne, 2015, https://kups.ub.uni-koeln.de/10364/

[14] M. A. DePristo, D. L. Hartl and D. M. Weinreich, Mutational Reversions During Adaptive Protein Evolution, Mol. Biol. Evol. 24 (2007), 1608-1610.

[15] J. A. G. M. de Visser, S.-C. Park and J. Krug, Exploring the effects of sex on empirical fitness landscapes, Am. Nat. 174 (2009), S15-S30.

[16] J. A. G. M. de Visser and J. Krug, Empirical fitness landscapes and the predictability of evolution, Nat. Rev. Genet. 15 (2014), 480-490.

[17] J. A. G. M. de Visser, S. F. Elena, I. Fragata and S. Matuszewski, The utility of fitness landscapes and big data for predicting evolution, Heredity 121 (2018), 401-405.

[18] F. Duque, A. Roldán-Correa and L. A. Valencia, Accessibility percolation with crossing valleys on n-ary trees, J. Stat. Phys. 174 (2019), 1027-1037.

[19] H. Flyvbjerg and B. Lautrup, Evolution in a rugged fitness landscape, Phys. Rev. A 46 (1992), 6714-6723.

[20] I. Fragata, A. Blanckaert, M. A. Dias Louro, D. A. Liberles and C. Bank, Evolution in the light of fitness landscape theory, Trends Ecol. Evol. 34 (2019), 69-82.

[21] J. Franke, G. Wergen and J. Krug, Records and sequences of records from random variables with a linear trend, J. Stat. Mech.: Theor. Exp. (2010), P10013.

[22] J. Franke, A. Klözer, J. A. G. M. de Visser and J. Krug, Evolutionary accessibility of mutational pathways, PLoS Comp. Biol. 7 (2011), e1002134.

[23] J. Franke and J. Krug, Evolutionary accessibility in tunably rugged fitness landscapes, J. Stat. Phys. 148 (2012), 706-723.

[24] S. Gavrilets and J. Gravner, Percolation on the hypercube and the evolution of reproductive isolation, J. Theor. Biol. 184 (1997), 51-65.

[25] J. H. Gillespie, Molecular evolution over the mutational landscape, Evolution 38 (1984), 1116-1129.

[26] P. Hegarty and A. Martinsson, On the existence of accessible paths in various models of fitness landscapes, Ann. Appl. Probab. 24 (2014), 1375-1395.

[27] S. Hwang, B. Schmiegelt, L. Ferretti and J. Krug, Universality classes of interaction structures for NK fitness landscapes, J. Stat. Phys. 172 (2018), 226-278.

[28] Y. Iwasa, F. Michor and M. A. Nowak, Stochastic Tunnels in Evolutionary Dynamics, Genetics 166 (2004), 1571-1579.

[29] K. Jain, J. Krug and S. C. Park, Evolutionary advantage of small populations on complex fitness 
landscapes, Evolution 65 (2011), 1945-1955.

[30] M. Josupeit, The influence of backwards mutations on the number of accessible paths in a fitness landscape, Bachelor thesis, University of Cologne, 2015, https://kups.ub.uni-koeln.de/10344/

[31] S. Kauffman and S. Levin, Towards a general theory of adaptive walks on rugged landscapes, $J$. Theor. Biol. 128 (1987), 11-45.

[32] S. A. Kauffman and E. D. Weinberger, The NK model of rugged fitness landscapes and its application to maturation of the immune response, J. Theor. Biol. 141 (1989), 211-245.

[33] S. A. Kauffman, The Origins of Order, Oxford University Press, London, 1993.

[34] R. Koekoek, P. Lesky and R. Swarttouw, Hypergeometric Orthogonal Polynomials and Their qAnalogues, Springer, Berlin, 2010.

[35] W. König, Branching processes in random environment. In: Probabilistic Structures in Evolution (eds. E. Baake and A. Wakolbinger), EMS Press, Berlin (2021), 23-41.

[36] L. Li, Phase transition for accessibility percolation on hypercubes, J. Theor. Probab. 31 (2018), 2072-2111.

[37] A. A. Louis, Contingency, convergence and hyper-astronomical numbers in biological evolution, Studies in History and Philosophy of Biological and Biomedical Science 58 (2016), 107-116.

[38] E. R. Lozovsky, T. Chookajorn, K. M. Brown, M. Imwong, P. J. Shaw, S. Kamchonwongpaisan, D. E. Neafsey, D. M. Weinreich and D. L. Hartl, Stepwise acquisition of pyrimethamine resistance in the malaria parasite, Proc. Natl. Acad. Sci. USA 106 (2009), 12025-12030.

[39] C. A. Macken, P. S. Hagan and A. S. Perelson, Evolutionary walks on rugged landscapes, SIAM J. Appl. Math. 51 (1991), 799-827.

[40] A. Martinsson, Accessibility percolation and first-passage site percolation on the unoriented binary hypercube, arXiv:1501.02206 (2015).

[41] A. Martinsson, Unoriented first-passage percolation on the n-cube, Ann. Appl. Probab. 26 (2016), $2597-2625$.

[42] A. Martinsson, First-passage percolation on Cartesian power graphs, Ann. Probab. 46 (2018), 1004-1041.

[43] J. Maynard Smith, Natural selection and the concept of a protein space, Nature 225 (1970), 563564.

[44] J. Neidhart and J. Krug, Adaptive walks and extreme value theory, Phys. Rev. Lett. 107 (2011), 178102.

[45] J. Neidhart, I. G. Szendro and J. Krug, Adaptation in tunably rugged fitness landscapes: the rough Mount Fuji model, Genetics 198 (2014), 699-721.

[46] S. Nowak and J. Krug, Accessibility percolation on n-trees, EPL 101 (2013), 66004.

[47] S. Nowak and J. Krug, Analysis of adaptive walks on NK fitness landscapes with different interaction schemes, J. Stat. Mech.: Theor. Exp. (2015), P06014.

[48] H. A. Orr, The population genetics of adaptation: The adaptation of DNA sequences, Evolution 56 (2002), 1317-1330.

[49] H. A. Orr, A minimum on the mean number of steps taken in adaptive walks, J. Theor. Biol. 220 (2003), 241-247.

[50] H. A. Orr, The population genetics of adaptation on correlated fitness landscapes: the block model, Evolution 60 (2006), 1113-1124.

[51] S. C. Park, I. G. Szendro, J. Neidhart and J. Krug, Phase transition in random adaptive walks on correlated fitness landscapes, Phys. Rev. E 91 (2015), 042707.

[52] S.-C. Park, J. Neidhart and J. Krug, Greedy adaptive walks on a correlated fitness landscape, J. Theor. Biol. 397 (2016), 89-102.

[53] S.-C. Park and J. Krug, $\delta$-exceedance records and random adaptive walks, J. Phys. A 49 (2016), 315601.

[54] A. S. Perelson and C. A. Macken, Protein evolution on partially correlated landscapes, Proc. Natl. Acad. Sci. USA 92 (1995), 9657-9661.

[55] F. J. Poelwijk, D. J. Kiviet, D. M. Weinreich and S. J. Tans, Empirical fitness landscapes reveal 
accessible evolutionary paths, Nature $\mathbf{4 4 5}$ (2007), 383-386.

[56] C. M. Reidys, Random Induced Subgraphs of Generalized n-Cubes, Adv. Appl. Math. 19 (1997), $360-377$.

[57] C. M. Reidys, Large components in random induced subgraphs of $n$-Cubes, Discr. Math. 309 (2009), 3113-3124.

[58] M. Roberts and L. Zhao, Increasing paths in regular trees, Electron. Commun. Probab. 18 (2013), $1-10$.

[59] B. Schmiegelt and J. Krug, Evolutionary accessibility of modular fitness landscapes, J. Stat. Phys. 154 (2014), 334-355.

[60] B. Schmiegelt, Sign epistasis networks, Master thesis, University of Cologne, 2016, https://kups.ub.uni-koeln.de/10398/

[61] B. Schmiegelt and J. Krug, Accessibility percolation on Cartesian power graphs, arXiv:1912.07925 (2019).

[62] P. F. Stadler, Fitness landscapes. In Biological Evolution and Statistical Physics, M. Lässig and A. Valleriani (eds.), Springer, Berlin, 2002, pp. 183-204.

[63] T. N. Starr and J. W. Thornton, Epistasis in protein evolution, Prot. Sci. 25 (2016), 1204-1218.

[64] I. G. Szendro, M. F. Schenk, J. Franke, J. Krug and J. A. G. M. de Visser, Quantitative analyses of empirical fitness landscapes, J. Stat. Mech.: Theor. Exp. (2013), P01005.

[65] I G. Szendro, J. Franke, J. A. G. M. de Visser and J. Krug, Predictability of evolution depends non-monotonically on population size, Proc. Natl. Acad. Sci. USA 110 (2013), 571-576.

[66] D. M. Weinreich and L. Chao, Rapid evolutionary escape of large populations from local fitness peaks is likely in nature, Evolution $\mathbf{5 9}$ (2005), 1175-1182.

[67] D. M. Weinreich, R. A. Watson and L. Chao, Perspective: Sign epistasis and genetic constraint on evolutionary trajectories, Evolution 59 (2005), 1165-1174.

[68] D. M. Weinreich, N. F. Delaney, M. A. DePristo and D. L. Hartl, Darwinian evolution can follow only very few mutational paths to fitter proteins, Science 312 (2006), 111-114.

[69] M. Zagorski, Z. Burda and B. Waclaw, Beyond the hypercube: Evolutionary accessibility of fitness landscapes with realistic mutational networks, PLoS Comp. Biol. 12 (2016), e1005218. 